\title{
Häufigkeit und Kosten von kognitiven Störungen in Deutschland
}

\author{
Occurence and Costs of Cognitive Disorders in Germany
}

Autoren

Institute
Steffi G. Riedel-Heller ${ }^{1}$, Hans-Helmut König ${ }^{2}$

Institut für Sozialmedizin, Arbeitsmedizin und Public Health (ISAP), Medizinische Fakultät, Universität Leipzig

Institut für Medizinische Soziologie, Sozialmedizin und Gesundheitsökonomie (IMSG), Universitätsklinikum Hamburg-Eppendorf

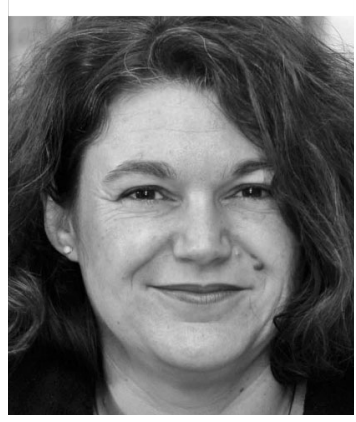

Prof. Dr. Steffi G. RiedelHeller

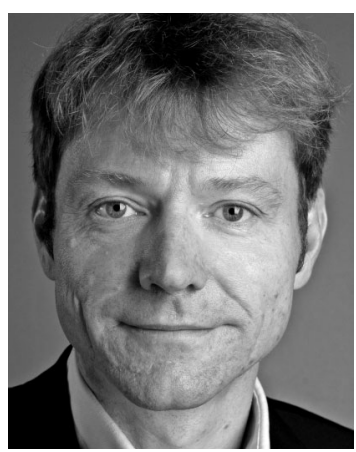

Prof. Dr. Hans-Helmut König

Bibliografie

Dol http://dx.doi.org/

10.1055/s-0031-1276937

Psychiat Prax 2011; 38 :

317-319

(c) Georg Thieme Verlag KG Stuttgart · New York .

ISSN 0303-4259

Korrespondenzadresse

Prof. Dr. med.

Steffi G. Riedel-Heller, MPH

Institut für Sozialmedizin,

Arbeitsmedizin und

Public Health (ISAP)

Philipp-Rosenthal-Straße 55

04103 Leipzig

Steffi.Riedel-Heller@medizin.

uni-leipzig.de

\section{Alternde Gesellschaft \\ $\nabla$}

Deutschland gehört zu den alternden Gesellschaften. Eine steigende durchschnittliche Lebenserwartung, eine konstant niedrige Geburtenrate und damit ein steigender Anteil älterer Menschen in der Gesellschaft charakterisieren die gegenwärtige demografische Entwicklung. Das statistische Bundesamt bezifferte 2010 die durchschnittliche Lebenserwartung neugeborener Jungen auf 77 Jahre und 4 Monate, für neugeborene Mädchen auf 82 Jahre und 6 Monate [1]. Auch ältere Menschen leben länger und altersassoziierte Erkrankungen nehmen zu. Mit der Aussicht auf ein langes Leben erhöht sich auch die Wahrscheinlichkeit von Erkrankungen, wie beispielsweise den Demenzerkrankungen. Man streitet noch darüber, ob im Durchschnitt der Preis für ein längeres Leben mit einer längeren von Krankheit und Gebrechen geprägten Phase zu bezahlen ist (sog. „extension of morbidity“), ob diese gleich bleibt und wir einfach länger gesund sind („dynamic equlibrium“) oder ob es gar im Zuge verschiedenster Verbesserungen in den Lebensbedingungen eine Kompression der durchschnittlich erwartbaren Krankheitsphase am Ende des Lebens gibt („compression of morbidity“). Obgleich einige Befunde sogar für letzteres Szenario sprechen [2], wird die Bedeutung altersassoziierter Erkrankungen wie der Demenzen zunehmen. Genaue Informationen dazu sind wichtig. Wie häufig sind aber Demenzen und leichte kognitive Störungen gegenwärtig in der deutschen Allgemeinbevölkerung?

\section{Häufigkeit kognitiver Störungen \\ $\nabla$}

Die Prävalenz reflektiert den Krankenstand in der Bevölkerung und ist vor allem für die Versorgungsplanung von Interesse. Für Deutschland liegen dazu nur wenige Studien vor. Erste Feldstudi- energebnisse publizierten Cooper et al. 1989 mit einer bevölkerungsbasierten Studie von über 65Jährigen in Mannheim $(n=617)$, in der entsprechend den ICD-9-Kriterien die Häufigkeit der Demenz mit 5,9\% angegeben wurde [3]. Fichter et al. 1995 führten eine Studie bei Hochbetagten durch $(85+, n=402)$, in der verschiedene Instrumente ${ }^{1}$ zum Einsatz kamen: Für die ICD-10 klinische Diagnose Demenz wurde bei den 85-89-Jährigen eine Prävalenz von $38,3 \%$ und bei den über 90 Jährigen von $57,3 \%$ berichtet [4-6]. Reischies publizierte 1997 Ergebnisse der Berliner Altersstudie und berichtete die Häufigkeit für Demenzerkrankungen nach DSM-III-R für verschiedene Altersgruppen (75-79: 9,3\%; 80-84: 9,3\%; 85-89: 18,6\%; 90+: 34,9\%) [7]. Die Leipziger Langzeitstudie in der Altenbevölkerung (LEILA75+) legt erstmals umfassende Ergebnisse auf der Grundlage einer großen bevölkerungsrepräsentativen Stichprobe von 1692 Senioren über 75 Jahre vor [8]. Die im Rahmen der Baseline-Erhebung der LEILA75+ ermittelte Prävalenz von Demenz in der Altenbevölkerung $75+$ betrug je nach den zugrunde liegenden Diagnosekriterien 12,4\% (ICD10) bis $17,4 \%$ (DSM-III-R) [9]. Die im Studienverlauf ermittelte jährliche Inzidenz (Neuerkrankungsrate) von Demenzen im Alter betrug 45,8 (ICD-10) bzw. 47,4 (DSM-III-R) pro 1000 Personen-Jahre [10]. Sowohl bezogen auf die Prävalenz als auch auf die Inzidenz zeigten sich erwartungsgemäß höhere Erkrankungsraten im höheren Alter. Die Ergebnisse korrespondieren recht gut mit denen anderer europäischer Feldstudien, obgleich es eine Variabilität gibt, die am ehesten auf methodische Gründe, wie der Fallidentifikation, der Falldefinition und des Umgangs der einzelnen Studien mit besonderen Herausforderungen von Feldstudien im hohen Alter (Mortalität, Hör- und

\footnotetext{
${ }^{1}$ GMS-AGECAT (85-89/90+ in \%): 23,4/31,4; ICD-10/SIDAM: 13,6/24.0; DSM-III-R/SIDAM: 23,6/40.2; DSMIII-R clinical: 38.3/57.3; ICD-10 clinical: 38.3/57.3.
} 
Sehbeeinträchtigungen, schwerste körperliche Erkrankungen) zurückzuführen ist.

Den Demenzerkrankungen gehen in der Regel leichte kognitive Störungen (MCI) voraus. Diese Risikogruppe ist insofern interessant, als hier mögliche Frühinterventionen im Sinne einer sekundären Prävention ansetzen könnten. In der LEILA75+ wurde bei etwa jeder fünften Person (19,3\%) ab 75 Jahren eine leichte kognitive Beeinträchtigung nach aktuellen $\mathrm{MCI}-K r i t e r i e n$ diagnostiziert $[11,12]$. Amnestische und nichtamnestische Subtypen waren mit $10 \%$ und 9,3\% hier ungefähr gleich häufig verteilt. Die im Studienverlauf ermittelte jährliche Inzidenz von $\mathrm{MCI}$ im Alter betrug 76,5 pro 1000 Personen-Jahre, wobei sich auch hier signifikant höhere Inzidenzraten in den höheren Altersgruppen zeigten. Von den Personen mit leichten kognitiven Beeinträchtigungen zur Baseline der LEILA75+ entwickelten 40,2\% eine klinisch manifeste Demenz innerhalb des Studienverlaufs (mittlere Beobachtungslänge $=4,3$ Jahre). Die Konversionsrate war damit deutlich höher als bei den kognitiv unbeeinträchtigen Teilnehmern (15\%) [13]. Hohe Konversionsraten zeigten sich insbesondere bei Personen mit amnestischen MCI-Subtypen.

Seit 2003 läuft die multizentrische AgeCoDe-Studie (German Study on Aging, Cognition and Dementia in Primary Care Patients) in Bonn, Düsseldorf, Hamburg, Leipzig, Mannheim und München. Sie ist strenggenommen keine bevölkerungsrepräsentative Studie, denn diese große Kohortenstudie $(n=3327$, Alter $75+)$ ist allgemeinarztbasiert. Da jedoch über 90\% der Senioren ihren Hausarzt regelmäßig aufsuchen, sind die Ergebnisse ein relativ gutes Abbild für die Situation in der Altenbevölkerung. Menschen in Pflegeheimen wurden im Rahmen der AgeCoDe-Studie jedoch nicht einbezogen. Bei 15,4\% der über 75-Jährigen (ohne Demenz) wurde eine leichte kognitive Beeinträchtigung nach aktuellen MCI-Kriterien diagnostiziert [14].

\section{Kosten von kognitiven Störungen}

Krankheitskostenstudien zielen auf die Beschreibung der Inanspruchnahme von medizinischen Leistungen und die Berechnung der krankheitsbedingten Kosten. Sie geben so Informationen zur ökonomischen Relevanz der Erkrankung für das Gesundheitssystem und die Gesellschaft.

Aktuell machen nur wenige deutsche Studien Aussagen zu den Kosten der Demenz. Hallauer und Kollegen schätzten die Gesamtkosten für eine demenzielle Erkrankung pro Patient und im Jahr 1998 auf $42800 €$ [15]. Die Berechnungen basierten auf Expertenbefragungen in verschiedenen Versorgungsbereichen. Aufgrund der einfachen Summierung unabhängiger Schätzer für formelle und informelle Pflege wurden in dieser Studie die Pflegekosten mit großer Wahrscheinlichkeit überschätzt. In einer auf Patientendaten basierenden Studie von Schulenburg und Kollegen wurden die Gesamtkosten für das Jahr 1995 auf 3594-5432 $€$ pro Patient für leichte Demenzen und auf 8375-17266 € für schwere Demenzen geschätzt [16]. Die Autoren inkludierten dabei ausschließlich Kosten der Kranken- und Pflegeversicherungen. Kosten der informellen Pflege, die von Familienangehörigen und Freunden getragen werden, wurden nicht berücksichtigt. Bei diesem Vorgehen wurden somit die informellen Kosten unterschätzt. Eine aktuelle Studie, die auf Daten der AgeCoDe-Studie beruht, hat den Ressourcenverbrauch und die Kosten von Demenz stadienspezifisch aus einer gesellschaftlichen Perspektive erfasst [17]. Dabei wurden auch die Kosten der informellen Pflege berücksichtigt. Die Kostenschätzung basiert auf 176 Demenz- fällen und 173 gematchten nicht dementen Kontrollen. Die jährlichen Gesamtkosten pro Patient für leichte Demenzen beliefen sich auf $15474 €$, für mittelschwere Demenzen auf $31551 €$ und für schwere Demenzen auf $41808 €$. Die Pflegekosten schlugen durchschnittlich mit ungefähr $3 / 4$ der Gesamtkosten zu Buche, die Hälfte davon resultierte aus der informellen Pflege durch Familie und Freunde, die andere Hälfte geht auf professionelle Pflege in Pflegeheimen, aber auch professionelle ambulante Pflegedienste zurück.

Deutlich weniger weiß man über die Kosten von leichten kognitiven Störungen. Hier liegt nur eine Pilotstudie vor, die die direkten Kosten von leichten kognitiven Störungen entsprechend aktueller Diagnosekriterien aus einer gesellschaftlichen Perspektive untersucht. Datengrundlage sind die im Leipziger Studienzentrum gesammelten Daten der AgeCoDe-Studie. Die durchschnittlichen jährlichen Kosten pro Patient mit MCI wurden auf $4443 €$ geschätzt und waren damit im Vergleich zu Senioren ohne kognitive Einschränkung, deren Kosten sich auf $3814 €$ beliefen, nicht signifikant höher [18]. Das ist nicht verwunderlich, wird doch nur ein kleiner Teil der Menschen mit kognitiven Störungen zum Beispiel im Hausarztsetting erkannt (12\%) [19]. Gleichwohl bleibt abzuwarten, ob mit den perspektivisch zu erwartenden Möglichkeiten für eine Frühintervention und Empfehlungen für eine frühe Behandlung sich hier Veränderungen ergeben werden.

\section{Implikation für Forschung und Praxis \\ $\nabla$}

Aktuell liegen belastbare Zahlen zur Häufigkeit demenzieller Erkrankungen in Deutschland vor, die sich recht gut mit internationalen Studien vergleichen lassen. Die epidemiologische Forschung konzentriert sich gegenwärtig auf die Beschreibung von Verläufen und deren Determinanten und auf die bessere Beschreibung von Risikopopulationen wie den leichten kognitiven Störungen. Trotz der substanziellen Konversionsraten ist mit einer MCI-Diagnose, die nach den gegenwärtigen am häufigsten angewandten Kriterien sich ausschließlich auf kognitive Leistungsmaße bezieht, eine Vorhersage für die Entwicklung einer Demenz im Einzelfall schwierig [11]. Immerhin bessert sich jeder Fünfte. Werden weitere Informationen einbezogen, wie etwa Daten zu der Beeinträchtigung von sog. instrumentellen Aktivitäten (IADL) des täglichen Lebens [20] und zum Krankheitsverlauf (längsschnittliches Risikosyndrom mit subjektiven Gedächtnisstörungen ohne kognitive Einschränkung, dann folgende $\mathrm{MCI}$, dann Demenz), wird die Vorhersagekraft deutlich besser [21]. Es geht also darum, Hochrisikogruppen für eine Konversion zu einer demenziellen Erkrankung besser zu beschreiben, um mögliche Interventionen, an denen mit Hochdruck gearbeitet wird, dieser Personengruppe zur Verfügung zu stellen. Hier könnte sich auch gerade die gestartete Leipziger Gesundheitsstudie LIFE (www. uni-leipzig-life.de), bei der 2000 über 65-jährige Leipziger Senioren untersucht werden, als vielversprechend erweisen; eine umfassende Geno- und Phänotypisierung unter Einsatz bildgebender Verfahren wird hier insbesondere Einblicke in die Gen-Umwelt-Interaktionen bei der Entstehung leichter kognitiver Beeinträchtigungen und Demenzen ermöglichen.

Auch für die Kosten von Demenzerkrankungen liegen für Deutschland jetzt verlässliche aktuelle stadienspezifische Daten vor. Die deutschen Studien haben gezeigt, dass der Erfassung der informellen Kosten, die insbesondere auf Pflegeleistungen der Angehörigen zurückzuführen sind, eine besondere Bedeutung zukommt. Zukünftig sollte in Längsschnittstudien untersucht 
werden, welche Faktoren die Kosten im Krankheitsverlauf beeinflussen, um Ansatzpunkte für kosteneffektive Interventionen zu finden.

Mit der demografischen Entwicklung wird nicht nur die Zahl der Demenzkranken zwangsläufig zunehmen. Für Deutschland geht eine Hochrechnung von einer Million Erkrankten im Jahr 2000 aus, die sich bis zum Jahr 2050 auf 2,3 Mio. Erkrankte erhöht [22]. Gleichzeitig ist von einer dramatischen Verschlechterung des Verhältnisses der arbeitsfähigen Personen, also den Personen die Demenzkranke pflegen oder für deren Pflege finanziell aufkommen, zu den an Demenz erkrankten Menschen auszugehen. Kommen derzeit auf jeden Demenzkranken 70 Erwerbstätige, werden es 2050 nur noch 21 Erwerbstätige sein [22]. Das wird Konsequenzen für die Pflegearrangements haben. Die Leipziger Langzeitstudie in der Altenbevölkerung konnte zeigen, dass es im Durchschnitt nur 2,9 Jahre dauert bis Senioren, die in einem Privathaushalt wohnen und an einer Demenz erkrankten, ins Heim eintreten [23]. Gleichwohl wollten die meisten Senioren zu Hause alt werden [24]. Dies macht deutlich, dass es neue Konzepte braucht, um den Herausforderungen alternder Gesellschaften zu begegnen. Wichtig ist eine gemeindenahe Versorgung mit zugehenden Formen der Betreuung. Darüber hinaus müssen Selbsthilfepotenziale der Angehörigen und der Gemeinde gestärkt werden, um eine Versorgung im Quartier sicherzustellen $[25,26]$.

\section{Literatur}

1 http://www.destatis.de/jetspeed/portal/cms/Sites/destatis/Internet/ DE/Presse/pm/2010/11/PD10_401_12621,templateld=renderPrint. psml

2 Doblhammer G, Kreft D. Live longer, suffer more? Trends in life expectancy and health. Bundesgesundheitsblatt Gesundheitsforschung Gesundheitsschutz 2011; 54: 907-914

3 Cooper B, Bickel H. Prävalenz und Inzidenz von Demenzerkrankungen in der Altenbevölkerung: Ergebnisse einer populationsbezogenen Längsschnittstudie in Mannheim. Nervenarzt 1989; 60: 472-482

4 Fichter MM, Meller I, Schröppel H et al. Dementia and cognitive impairment in the oldest old in the community. Prevalence and comorbidity. Br J Psychiatry 1995; 166: 621-629

5 Meller I, Fichter M, Schröppel H. Incidence of depression in octo- and nongenerians: results of an epidemiological follow-up community study. Eur Arch Psychiatry Clin Neurosci 1996; 246: 93-99

6 Meller I, Fichter M, Schröppel H et al. Mental and somatic health and need for care in octo- and nonagenerians. An epidemiological community study. Eur Arch Psychiatry Clin Neurosci 1993; 242: 286-292

7 Reischies FM, Geiselmann B, Gessner R et al. Demenz bei Hochbetagten. Ergebnisse der Berliner Altersstudie. Nervenarzt 1997; 68: 719-729

8 Riedel-Heller SG, Schork A, Matschinger $\mathrm{H}$ et al. Recruitment procedures and their impact on the prevalence of dementia. Results from the Leipzig Longitudinal Study of the Aged LEILA75+). Neuroepidemiology 2000; 19 : $130-140$
9 Riedel-Heller SG, Busse A, Aurich C et al. Prevalence of dementia according to DSM-III-R and ICD-10: results of the Leipzig Longitudinal Study of the Aged (LEILA75+) Part 1. Br J Psychiatry 2001; 179: 250-254

10 Riedel-Heller SG, Busse A, Aurich C et al. Incidence of dementia according to DSM-III-R and ICD-10: results of the Leipzig Longitudinal Study of the Aged (LEILA75+), Part 2. Br J Psychiatry 2001; 179: 255-260

11 Winblad B, Palmer K, Kivipelto $M$ et al. Mild cognitive impairment beyond controversies, towards a consensus: report of the International Working Group on Mild Cognitive Impairment. J Intern Med 2004; 256: $240-246$

12 Busse A, Hensel A, Gühne U et al. Mild cognitive impairment. Long-term course of four clinical subtypes. Neurology 2006; 67: 2176-2185

13 Luck T, Busse A, Hensel A et al. Leichte kognitive Beeinträchtigungen und Demenzentwicklung. Psychiat Prax 2008; 35: 331-336

14 Luck T, Riedel-Heller SG, Kaduszkiewicz H et al., AgeCoDe group. Mild cognitive impairment in general practice: age-specific prevalence and correlate results from the German study on ageing, cognition and dementia in primary care patients (AgeCoDe). Dement Geriatr Cogn Disord 2007; $24: 307-16$

15 Hallauer JF, Schons M, Smala A et al. Untersuchung von Krankheitskosten bei Patienten mit Alzheimer-Erkrankung in Deutschland. Gesundh ökon Qual manag 2000; 5: 73-79

16 Schulenburg JM, Schulenburg I, Horn R et al. Cost of treatment and care of Alzheimer's disease in Germany. In: Jönsson B, Karlsson G, Winblad B, eds. Health Economics of Dementia. Chichester: John Wiley \& Sons; 1998: $217-230$

17 Leicht H, Heinrich S, Heider D et al., for the AgeCoDe study group. Net costs of dementia by disease stage. Acta Psychiatr Scand 2011, e-first: DOI: $10.1111 / \mathrm{j} .1600-0447.2011 .01741 . x$

18 Luppa M, Heinrich S, Matschinger $H$ et al. Direct costs associated with mild cognitive impairment in primary care. Int J Geriatr Psychiatry 2008; 23: 963-971

19 Kaduszkiewicz H, Zimmermann T, van den Bussche $H$ et al., AgeCoDe Study Group. Do general practitioners recognize mild cognitive impairment in their patients? J Nutr Health Aging 2010; 14: 697-702

20 Luck T, Luppa M, Angermeyer MC et al. Impact of impairment in instrumental activities of daily living and mild cognitive impairment on time to incident dementia: results of the Leipzig Longitudinal Study of the Aged. Psychol Med 2011; 41: 1087-1097

21 Jessen F, Wiese B, Bachmann C et al., German Study on Aging, Cognition and Dementia in Primary Care Patients Study Group. Prediction of dementia by subjective memory impairment: effects of severity and temporal association with cognitive impairment. Arch Gen Psychiatry 2010; 67: 414-422

22 Wancata J, Musalek M, Alexandrowicz R et al. Number of dementia sufferers in Europe between the years 2000 and 2050. Eur Psychiatry 2003; 18: 306-313

23 Luck T, Luppa $M$, Weber $S$ et al. Time until Institutionalization in Incident Dementia Cases - Results of the Leipzig Longitudinal Study of the Aged (LEILA 75+). Neuroepidemiology 2008; 31: 100-108

24 Fleischer S, Roling G, Beutner K et al. Growing old at home - a randomized controlled trial to investigate the effectiveness and cost-effectiveness of preventive home visits to reduce nursing home admissions: study protocol [NCT00644826]. BMC Public Health 2008; 28: 185

25 Riedel-Heller SG. Sozialpsychiatrie als Forschungsfeld - Antworten auf die Fragen von morgen. Neuropsychiatr 2009; 23: 249-252

26 Wancata J, Meise U. Soziotherapeutische Interventionen - ausreichend genützt? Neuropsychiatr 2010; 24: 221-223 